\title{
Investigating the Effect of Piezoelectric Polarization on GaN-Based LEDs with Different Prestrain Layer by Temperature-Dependent Electroluminescence
}

\author{
C. K. Wang, ${ }^{1}$ Y. Z. Chiou, ${ }^{1}$ T. H. Chiang, ${ }^{2}$ and T. K. Lin ${ }^{2}$ \\ ${ }^{1}$ Department of Electronic Engineering, Southern Taiwan University of Science and Technology, Tainan 71005, Taiwan \\ ${ }^{2}$ Epistar Corporation, Tainan 744, Taiwan \\ Correspondence should be addressed to C. K. Wang; ckwang@mail.stust.edu.tw
}

Received 24 July 2014; Revised 11 September 2014; Accepted 16 September 2014

Academic Editor: Sheng-Po Chang

Copyright (C) 2015 C. K. Wang et al. This is an open access article distributed under the Creative Commons Attribution License, which permits unrestricted use, distribution, and reproduction in any medium, provided the original work is properly cited.

\begin{abstract}
The effect of piezoelectric polarization on GaN-based light emitting diodes (LEDs) with different kinds of prestrain layers between the multiple quantum wells (MQWs) and n-GaN layer is studied and demonstrated. Compared with the conventional LED, more than $10 \%$ enhancement in the output power of the LED with prestrain layer can be attributed to the reduction of polarization field within MQWs region. In this study, we reported a simple method to provide useful comparison of polarization fields within active region in GaN-based LEDs by using temperature-dependent electroluminescence (EL) measurement. The results pointed out that the polarization field of conventional LED was stronger than that of the others due to larger variation of the wavelength transition position (i.e., blue-shift change to red-shift) from 300 to $350 \mathrm{~K}$, and thus the larger polarization field must be effectively screened by injecting more carriers into the MQWs region.
\end{abstract}

\section{Introduction}

GaN-based materials generally exist in a wurtzite crystal structure which has a strong polarization field along the $c$-plane direction. Therefore, the space charges induced by spontaneous and piezoelectric polarization fields were at the interfaces of heterostructure for GaN-based materials, for example, in the $c$-plane In GaN/GaN multiple quantum wells (MQWs) structure. This inherent effect, which was called quantum-confined stark effect (QCSE), has resulted in the reduction of quantum efficiency for the GaN-based LEDs. This is because the QCSE within MQWs region lead to the significant spatial separation of the electron and hole wave functions and the reduction of the electron-hole recombination probability [1-5]. Furthermore, investigating the spontaneous and piezoelectric polarization-induced electrostatic field on InGaN/GaN MQWs heterostructure is a very important key issue currently. Thus, many novel and useful growth techniques and structures have been developed to release the strain and reduce the separation of the wave function in the InGaN QWs to improve the optical properties of nitride-based LEDs [6-14].
Besides, some research groups also calculate both the internal electric-field strengths and the carrier density screening of the potential by the measured spectral shifts using photoluminescence (PL) system [15-18]. In our previous work [19], we also successively reported a simple method by temperature-dependent EL measurement to provide useful comparison of electrostatic fields within the QWs of GaN-based LEDs, specifically for structures consisting of identical active regions with different barrier thicknesses. On the other hand, several studies have revealed that the optical properties of LEDs with the structures of InGaN/GaN shortperiod superlattices (SPS) or low-temperature (LT) GaN layer between the $\mathrm{n}-\mathrm{GaN}$ and MQWs can be improved due to the release of the residual strain in MQWs, reduction of the QCSE, enhancement of electron-hole recombination rate, reduction of the $\mathrm{V}$-defect density, and improvement of the crystal quality in MQWs $[17,18,20,21]$. However, it is very necessary to investigate the more detailed relationship between prestrain layer and QCSE. Moreover, in this study, the strengths of the polarization field and the effect of QCSE in the active region of GaN-based LEDs with different 


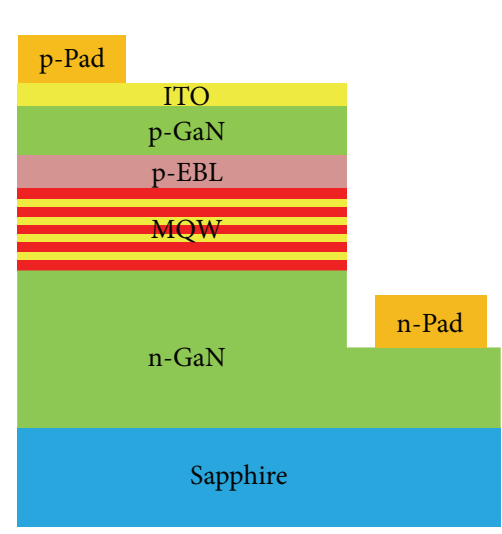

(a) LED I

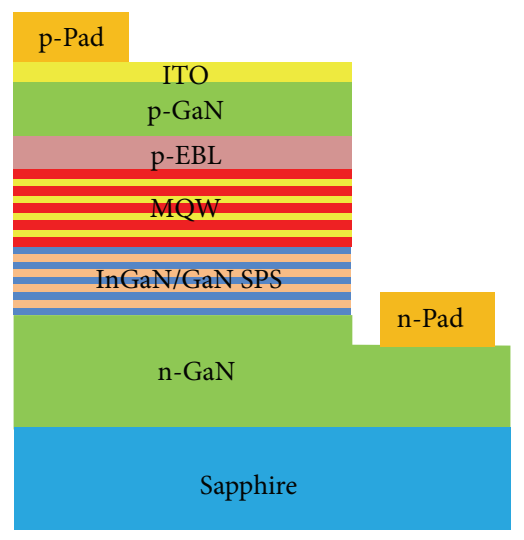

(b) LED II

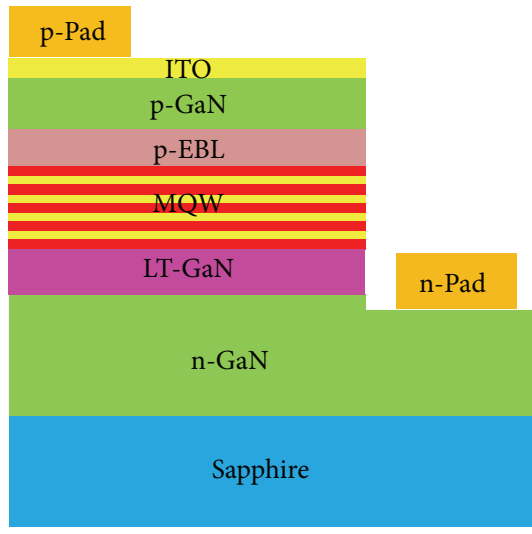

(c) LED III

FIGURE 1: The schematic structures of the three fabricated LEDs. (a) LED I without any specific prestrain interlayer. (b) LED II with InGaN/GaN SPS interlayer. (c) LED III with LT-GaN interlayer.

prestrain layer were compared by using our previous method of temperature-dependent EL measurement.

\section{Experimental}

All samples used in this study were grown on $c$-plane (0001) sapphire $\left(\mathrm{Al}_{2} \mathrm{O}_{3}\right)$ substrate by metal-organic chemical vapor deposition (MOCVD). During the growth, trimethylgallium (TMGa), trimethylindium (TMIn), and ammonia $\left(\mathrm{NH}_{3}\right)$ were used as gallium, indium, and nitrogen sources, respectively, while biscyclopentadienyl magnesium $\left(\mathrm{CP}_{2} \mathrm{Mg}\right)$ and disilane $\left(\mathrm{Si}_{2} \mathrm{H}_{6}\right)$ were used as the p-type and n-type doping sources, respectively. The conventional LED structure, called LED I, consists of a $30 \mathrm{~nm}$ thick buffer layer grown at low temperature, a $1.5 \mu \mathrm{m}$ thick undoped $\mathrm{GaN}$ layer grown at $1040^{\circ} \mathrm{C}$, a $2 \mu \mathrm{m}$ thick Si-doped n-GaN layer grown at $1040^{\circ} \mathrm{C}$, and an MQW active region grown at $750^{\circ} \mathrm{C}$. The MQW active region consists of 10 periods of $3 \mathrm{~nm}$ thick InGaN well layers (with nominal indium content $\sim 17 \%$ ) and $10 \mathrm{~nm}$ thick GaN barrier layers. Subsequently, the temperature was elevated to $1000^{\circ} \mathrm{C}$ to grow a $30 \mathrm{~nm}$ thick $\mathrm{Mg}$-doped $\mathrm{p}-\mathrm{Al}_{0.15} \mathrm{Ga}_{0.85} \mathrm{~N}$ electron blocking layer (EBL) and a $0.3 \mu \mathrm{m}$ thick p-GaN layer. In order to reduce the strain and polarization effect in the active region, the LEDs with prestrain interlayer structure are also prepared. As shown in Figure 1, the LEDs with lowerindium content ( $5 \%)$ 10-pair InGaN ( $2 \mathrm{~nm}) / \mathrm{GaN}(3 \mathrm{~nm})$ SPS interlayer grown at $800^{\circ} \mathrm{C}$ were labeled as LED II. And the LEDs with LT-GaN $(50 \mathrm{~nm})$ interlayer grown at $750^{\circ} \mathrm{C}$ were labeled as LED III. The as-grown samples were subsequently annealed at $750^{\circ} \mathrm{C}$ in $\mathrm{N}_{2}$ ambient to active $\mathrm{Mg}$ in the p-type layers.

After the growth, the surface of the samples was partially etched until the $2 \mu \mathrm{m}$ thick Si-doped n-GaN layer was exposed. Subsequently, a $70 \mathrm{~nm}$ thick indium-tin oxide (ITO) layer was deposited by sputter system onto the $\mathrm{p}-\mathrm{GaN}$ layer to serve as a current spreading layer. $\mathrm{A} \mathrm{Ni} / \mathrm{Au}(30 \mathrm{~nm} / 500 \mathrm{~nm})$ metal contact was deposited on the ITO layer to form the p-electrode. A Ti/Al/Ti/Au $(15 \mathrm{~nm} / 450 \mathrm{~nm} / 50 \mathrm{~nm} / 500 \mathrm{~nm})$

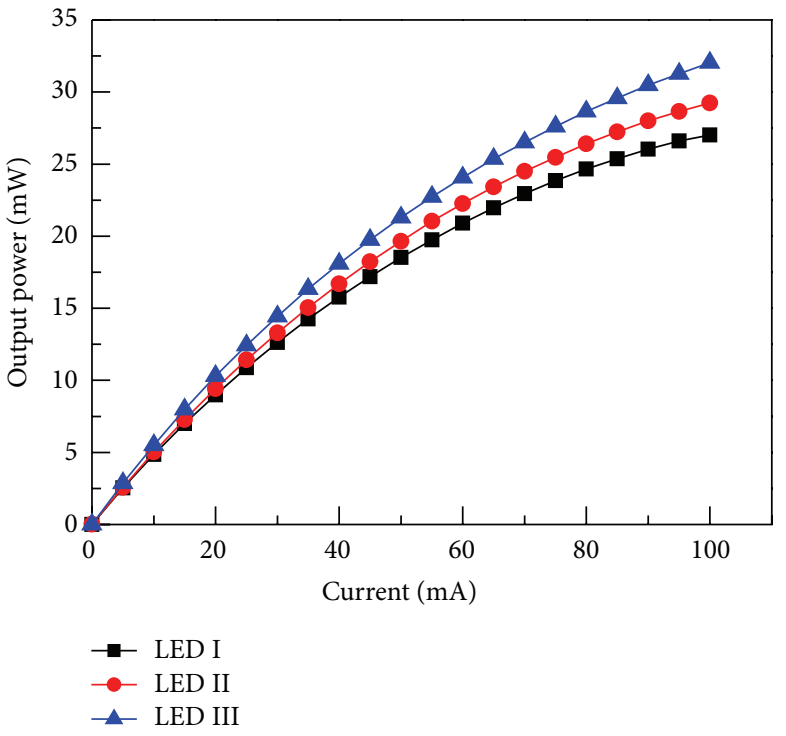

FIGURE 2: Light output powers measured from LEDs I, II, and III as functions of injection currents.

metal contact was then deposited on the exposed n-GaN layer to form the n-electrode. The LEDs fabricated in this study had a chip size of $250 \times 580 \mu \mathrm{m}^{2}$. The light output powers were measured by the integrating sphere detector. The optical characteristics of these samples were measured by EL system.

\section{Results and Discussion}

Figure 2 shows the measured light output power as a function of the injection current for these three fabricated LED structures. In order to avoid the effects of self-heating, we applied pulse-width-modulated injection currents on each of the measured LEDs [22]. By increasing the injection current, the light output powers of these three fabricated LED 


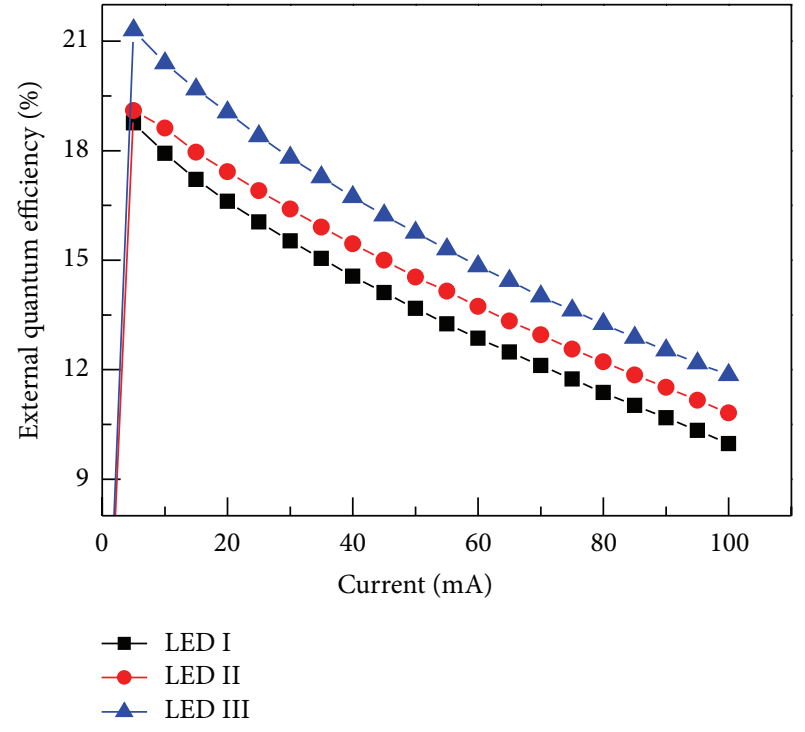

FIGURE 3: EQE as functions of injection currents for LEDs I, II, and III.

structures are increased. At an injection current of $20 \mathrm{~mA}$, the light output powers of LEDs I, II, and III are 8.99, 9.42, and $10.30 \mathrm{~mW}$, respectively. The output powers of LEDs II and III with prestrain structures were 4.8 and $14.6 \%$ higher than those of LED I, respectively. It was also found that the difference of output power between these three LEDs was remarkably enlarged especially at higher injection current. Compared with LED I, the light output powers of LEDs II and III were improved by approximately $8.2 \%$ and $18.6 \%$ under an injection current of $100 \mathrm{~mA}$, respectively. These results show that the light output power can be improved obviously with inserting the prestrain interlayers between the $\mathrm{n}-\mathrm{GaN}$ and MQWs. This means that the improvement of output power can be attributed to the release of the residual strain, the reduction of the polarization field and QCSE, the enhancement of electron-hole recombination rate, the reduction the $\mathrm{V}$-defect density, and the improvement of the crystal quality in MQWs for LEDs II and III [20, 21]. Figure 3 shows the measured external quantum efficiency (EQE) as a function of injection current. The EQE is defined as

$$
\mathrm{EQE}=\frac{P /(h v)}{I / e}
$$

where $P$ is the light output power, $h v$ is the energy of photon emitted from a semiconductor, $I$ is the injection current, and $e$ is the electron charge. When the LED is operated with injection current, the light power and emission wavelength are measured by integrating sphere detector at the same time. Thus, the values of EQE can be calculated by using (1). The peak efficiency of LEDs I, II, and III is $18.8 \%, 19.1 \%$, and $23.3 \%$, respectively. The improvement of EQE for LEDs II and III is mainly due to the partial strain relaxation within the active region, which resulted in a less tilt of band-edge and a better overlap of the electron and hole wave functions in each quantum well. It implies that the polarization field within the active region in this study could be reduced effectively by inserting prestrain interlayer structure.

In order to further differentiate the piezoelectric polarization field for these three fabricated LED structures, the emission spectrum characteristics of the LEDs by temperaturedependent EL were measured and achieved. The temperature of the LEDs was varied by using a heat controller. The temperature was ramped from $300 \mathrm{~K}$ up to $350 \mathrm{~K}$ with a step of $10 \mathrm{~K}$. Figures 4(a)-4(c) show the peak emission wavelength of these three fabricated LEDs at various ambient temperatures as a function of injection current. First, it was obviously found that the overall emission wavelength in these three LEDs shifted to longer wavelength as the ambient temperature increased. The temperature dependence of the energy band-gap for a semiconductor can be expressed by the Varshni formula:

$$
E_{g}=\left.E_{g}\right|_{T=0 \mathrm{~K}}-\frac{\alpha T^{2}}{T+\beta},
$$

where $\alpha$ and $\beta$ are fitting parameters, frequently called the Varshni parameters [23]. It indicates that the energy bandgap of semiconductor generally decreases as the temperature increases, and thus the overall emission wavelength of the LEDs shifted to longer wavelength. The reason is that energy band-gap of quantum well became narrow which is caused by the increase in ambient temperature according to the Varshni effect $[23,24]$.

Then, it was also found that the wavelength of these three LEDs initially shifted toward shorter wavelength and then shifted toward longer wavelength as the injection current was increased. It is well known that the phenomenon of the wavelength shifting toward shorter wavelength was called blue-shift, which can be attributed to the screening effect and band-filling effect [25]. On the contrary, the wavelength shifted toward longer wavelength was called red-shift, which was induced by the thermal effect created by the parasitic resistances of contacts and semiconductor layers due to the $I^{2} R$ dependence of Joule heating [24]. Regarding the blueshift values of emission wavelength which was related with polarization field within the active region, it was found that these values for LEDs I, II, and III at room temperature were $1.37,1.22$, and $1.11 \mathrm{~nm}$, respectively. Such a result showed that the conventional LEDs possess a larger blue-shift value than that of LEDs II and III with prestrain interlayer. This is because the stronger QCSE for LED I induced by the spontaneous and piezoelectric fields in the MQW layers would show an obvious screening effect and band-filling effect. These results once more explained that the polarization field within the active region in this study could be reduced effectively by inserting prestrain interlayer structure. Therefore, the output powers of LEDs II and III were improved due to a better overlap of the electron and hole wave functions in each quantum well.

On the other hand, it is very worthy to note that the value of injection current, at which the wavelength of blueshift changed to red-shift, shifted to larger injection current 


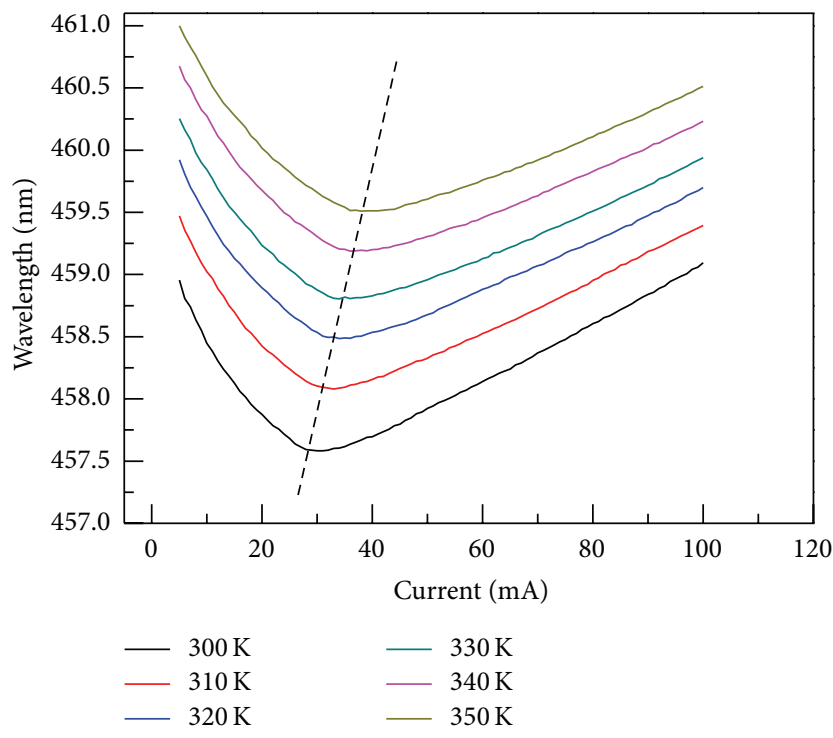

(a) LED I

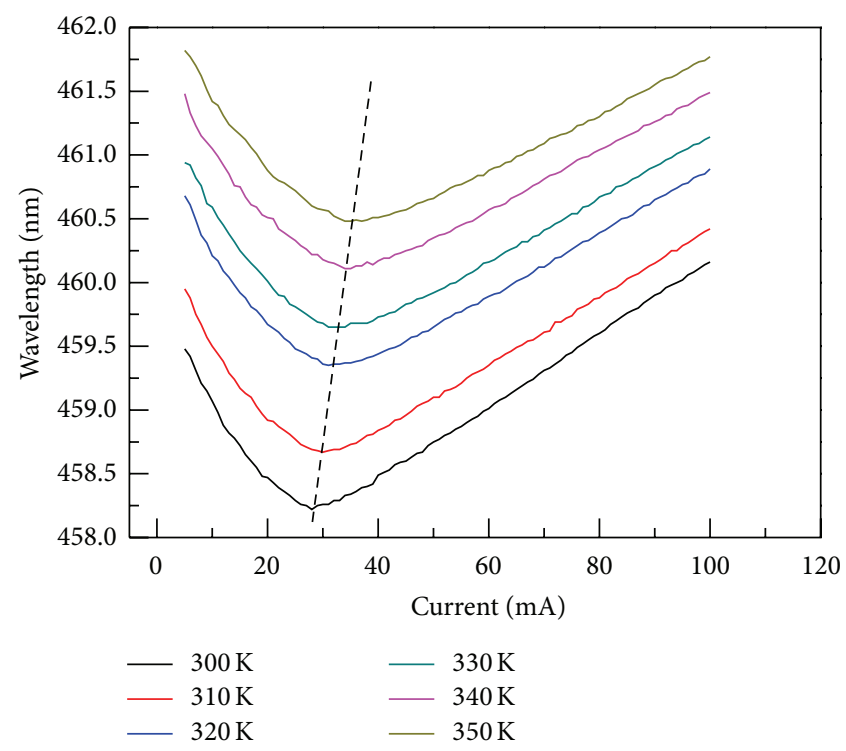

(b) LED II

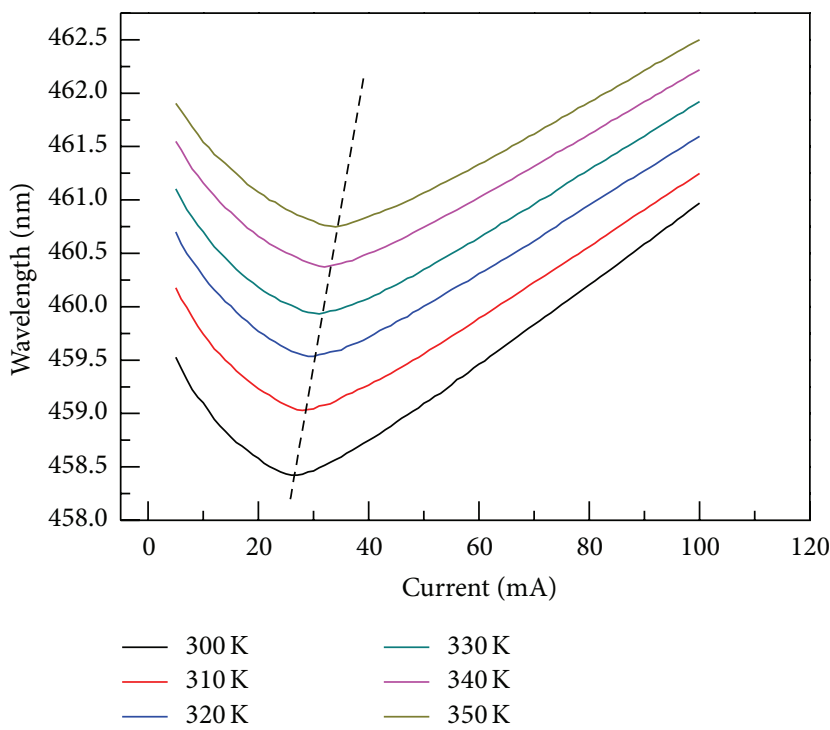

(c) LED III

Figure 4: The emission wavelengths as functions of injection currents under different ambient temperatures measured from 300 to $350 \mathrm{~K}$ for (a) LED I, (b) LED II, and (c) LED III.

when the temperature increased, as shown in the dashed line of Figure 4. It can be seen clearly that LED I as shown in Figure 4(a) has a more obvious change than those of LEDs II and III as shown in Figures 4(b) and 4(c). This is because the carriers injected into the MQWs would be excited by the higher ambient temperature. Thus, these excited carriers would escape from the MQWs, and the energy bandgap of MQWs caused by QCSE cannot be screened and filled effectively. In other words, it needs higher injection current to provide more carriers which can complete the screening effect of LEDs. Therefore, LED I without prestrain structure with more serious QCSE resulting from larger polarization field within the active region must inject more currents into QWs to compensate the screening effect.
Figure 5 also shows the blue-shift value as a function of ambient temperature on these three fabricated LED structures. It was found that the blue-shift values of these three LEDs kept almost the same with increasing ambient temperature. Such a result indicated that the value of blueshift induced by screening and band-filling effect for these three LEDs was independent of the ambient temperature. In other words, this pointed out that QCSE caused by polarization field in this study did not change with the ambient temperature increase. Figure 6 shows the variation of the injection current of wavelength transition position from blue-shift to red-shift, which was extracted from the dashed line of Figure 4, as a function of the ambient temperature. It can be seen clearly that the slope of conventional LED is 


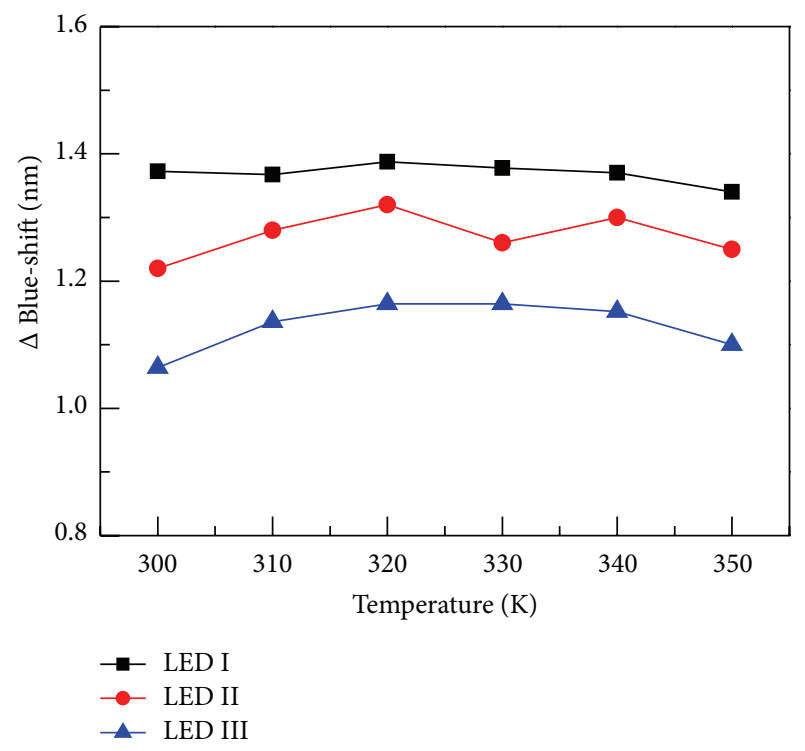

FIGURE 5: Blue-shift values of emission wavelength as functions of ambient temperatures for the three fabricated LEDs.

larger than that of the other LEDs with prestrain interlayer. According to Figure 6, it was found that the injection current of wavelength transition position from blue-shift to red-shift of LED I, LED II, and LED III is 30,28 , and $27 \mathrm{~mA}$ at room temperature and that those of LED I, LED II, and LED III are 40,36 , and $34 \mathrm{~mA}$ at $350 \mathrm{~K}$. The results again imply that the polarization fields within the active region of LED I were stronger than others due to larger variation of the injection current of the wavelength transition position from $300 \mathrm{~K}$ to $350 \mathrm{~K}$, and thus it needed more injection carriers (higher injection current) to complete the screening of QCSE within the active region. Thus, we reported a simple method to provide useful comparison of electrostatic fields within the InGaN MQW active region on the GaN-based LEDs, specifically for structures consisting of identical active regions with different prestrain layers.

\section{Conclusions}

The effect of piezoelectric polarization on GaN-based LEDs with different kinds of prestrain layers between the MQWs layers and $\mathrm{n}-\mathrm{GaN}$ layer is studied and demonstrated. Compared with the conventional LED, it was found that more than $10 \%$ enhancement in the output power of the LED with prestrain layer can be attributed to the reduction of polarization field within MQWs layers. In this study, we reported a simple method to provide useful comparison of polarization fields in the LEDs which were estimated using temperature-dependent EL measurement. The results pointed out that the polarization field of conventional LED was stronger than that of the others due to larger variation of the wavelength transition position (i.e., blue-shift change to red-shift) from 300 to $350 \mathrm{~K}$, and thus the larger polarization field must be effectively screened by injecting more carriers.

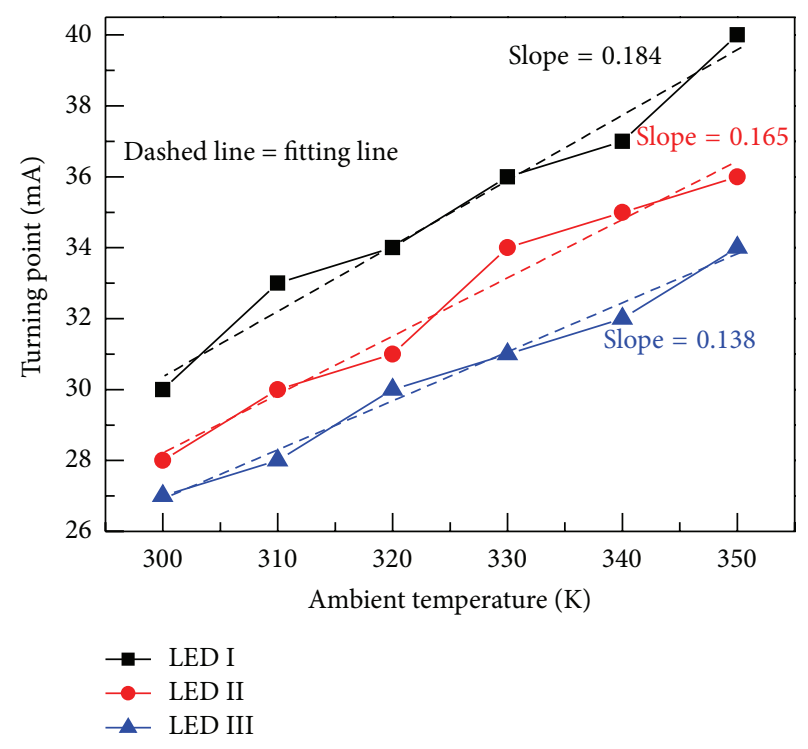

FIGURE 6: The turning points of injection currents (i.e., wavelength transition position from blue-shift to red-shift as shown by the dashed lines in Figures 4(a)-4(c)) as functions of ambient temperatures for the three fabricated LEDs.

\section{Conflict of Interests}

The authors declare that there is no conflict of interests regarding the publication of this paper.

\section{Acknowledgment}

This work was supported by the National Science Council of Taiwan under Contract nos. NSC 101-2221-E-218-023-MY2 and NSC 101-2632-E-218-001-MY3.

\section{References}

[1] Z. H. Zhang, W. Liu, Z. Ju et al., "Self-screening of the quantum confined Stark effect by the polarization induced bulk charges in the quantum barriers," Applied Physics Letters, vol. 104, Article ID 243501, 2014.

[2] A. Dadgar, L. Groh, S. Metzner et al., "Green to blue polarization compensated c-axis oriented multi-quantum wells by AlGaInN barrier layers," Applied Physics Letters, vol. 102, no. 6, Article ID 062110, 2013.

[3] T. Langer, H. Jönen, A. Kruse, H. Bremers, U. Rossow, and A. Hangleiter, "Strain-induced defects as nonradiative recombination centers in green-emitting GaInN/GaN quantum well structures," Applied Physics Letters, vol. 103, no. 2, Article ID 022108, 2013.

[4] M. Thomsen, H. Jönen, U. Rossow, and A. Hangleiter, "Spontaneous polarization field in polar and nonpolar GaInN/GaN quantum well structures," Physica Status Solidi (B) Basic Research, vol. 248, no. 3, pp. 627-631, 2011.

[5] H.-M. Huang, T.-C. Lu, C.-Y. Chang et al., "Investigation of emission polarization and strain in InGaN-GaN multiple quantum wells on nanorod epitaxially lateral overgrowth templates," Journal of Lightwave Technology, vol. 29, no. 18, Article ID 5983376, pp. 2761-2765, 2011. 
[6] H. Zhao, G. Liu, and N. Tansu, "Analysis of InGaN-delta-InN quantum wells for light-emitting diodes," Applied Physics Letters, vol. 97, no. 13, Article ID 131114, 2010.

[7] C.-T. Liao, M.-C. Tsai, B.-T. Liou, S.-H. Yen, and Y.-K. Kuo, "Improvement in output power of a $460 \mathrm{~nm}$ InGaN lightemitting diode using staggered quantum well," Journal of Applied Physics, vol. 108, no. 6, Article ID 063107, 2010.

[8] J. Park and Y. Kawakami, "Photoluminescence property of InGaN single quantum well with embedded AlGaN $\delta$ layer," Applied Physics Letters, vol. 88, no. 20, Article ID 202107, 2006.

[9] Y. Li, B. Liu, R. Zhang, Z. Xie, and Y. Zheng, "Investigation of optical properties of InGaNInNInGaN/GaN quantum-well in the green spectral regime," Physica E, vol. 44, p. 821, 2012.

[10] L. Zhang, K. Cheng, H. Liang, R. Lieten, M. Leys, and G. Borghs, "Photoluminescence studies of polarization effects in InGaN/(In)GaN multiple quantum well structures," Japanese Journal of Applied Physics, vol. 51, no. 3, Article ID 030207, 2012.

[11] H. Zhao, R. A. Arif, Y.-K. Ee, and N. Tansu, "Self-cnsistent analysis of strain-compensated InGaN-AlGaN quantum wells for lasers and light-emitting diodes," IEEE Journal of Quantum Electronics, vol. 45, no. 1, pp. 66-78, 2009.

[12] C.-L. Tsai, G.-C. Fan, and Y.-S. Lee, "Effects of strain-compensated AlGaN/InGaN superlattice barriers on the optical properties of InGaN light-emitting diodes," Applied Physics A: Materials Science and Processing, vol. 104, no. 1, pp. 319-323, 2011.

[13] C. H. Wang, S. P. Chang, P. H. Ku et al., "Hole transport improvement in InGaN/GaN light-emitting diodes by gradedcomposition multiple quantum barriers," Applied Physics Letters, vol. 99, no. 17, Article ID 171106, 2011.

[14] R. M. Farrell, E. C. Young, F. Wu, S. P. Denbaars, and J. S. Speck, "Materials and growth issues for high-performance nonpolar and semipolar light-emitting devices," Semiconductor Science and Technology, vol. 27, no. 2, Article ID 024001, 2012.

[15] V. Ramesh, A. Kikuchi, K. Kishino, M. Funato, and Y. Kawakami, "Strain relaxation effect by nanotexturing InGaN/GaN multiple quantum well," Journal of Applied Physics, vol. 107, no. 11, Article ID 114303, 2010.

[16] J. Xu, M. F. Schubert, D. Zhu et al., "Effects of polarization-field tuning in GaInN light-emitting diodes," Applied Physics Letters, vol. 99, no. 4, Article ID 041105, 2011.

[17] S. P. Chang, C. H. Wang, C. H. Chiu et al., "Characteristics of efficiency droop in GaN-based light emitting diodes with an insertion layer between the multiple quantum wells and $\mathrm{n}-\mathrm{GaN}$ layer," Applied Physics Letters, vol. 97, no. 25, Article ID 251114, 2010.

[18] C.-F. Huang, C.-Y. Chen, C.-F. Lu, and C. C. Yang, "Reduced injection current induced blueshift in an InGaNGaN quantumwell light-emitting diode of prestrained growth," Applied Physics Letters, vol. 91, no. 5, Article ID 051121, 2007.

[19] C. K. Wang, T. H. Chiang, K. Y. Chen et al., "Investigating the effect of piezoelectric polarization on GaN-based LEDs with different quantum barrier thickness," Journal of Display Technology, vol. 9, no. 4, pp. 207-212, 2013.

[20] S. J. Leem, Y. C. Shin, K. C. Kim et al., "The effect of the low-mole InGaN structure and InGaN/GaN strained layer superlattices on optical performance of multiple quantum well active layers," Journal of Crystal Growth, vol. 311, no. 1, pp. 103-106, 2008.

[21] R.-M. Lin, Y.-H. Lin, C.-H. Chiang et al., "Inserting a lowtemperature $\mathrm{n}-\mathrm{GaN}$ underlying layer to separate nonradiative recombination centers improves the luminescence efficiency of blue InGaN/GaN LEDs," Microelectronics Reliability, vol. 50, no. 5, pp. 679-682, 2010.

[22] D. H. Lee, H. K. Lee, J. S. Yu et al., “Temperature and thermal characteristics of InGaN/GaN vertical light-emitting diodes on electroplated copper," Semiconductor Science and Technology, vol. 26, no. 5, Article ID 055014, 2011.

[23] Y. P. Varshni, "Temperature dependence of the energy gap in semiconductors," Physica, vol. 34, no. 1, pp. 149-154, 1967.

[24] E. F. Schubert, Light-Emitting Diodes, Cambridge University Press, Cambridge, Mass, USA, 2nd edition, 2006.

[25] D.-Y. Lee, S.-H. Han, D.-J. Lee et al., "Effect of an electron blocking layer on the piezoelectric field in InGaN/GaN multiple quantum well light-emitting diodes," Applied Physics Letters, vol. 100, no. 4, Article ID 041119, 2012. 

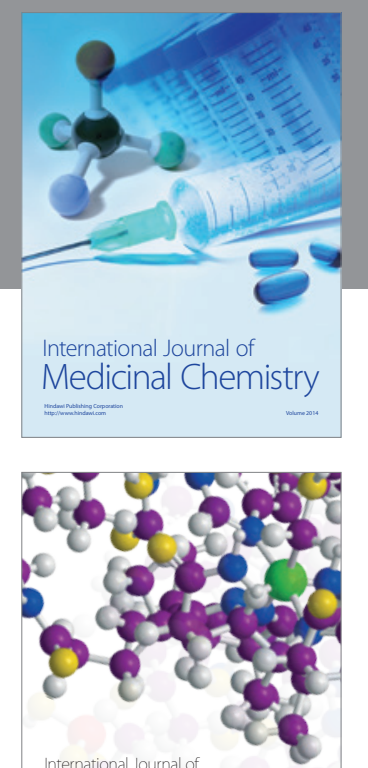

\section{Carbohydrate} Chemistry

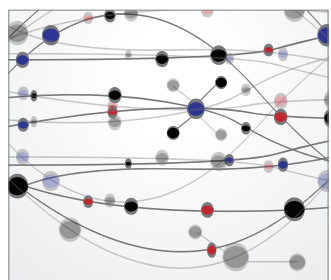

The Scientific World Journal
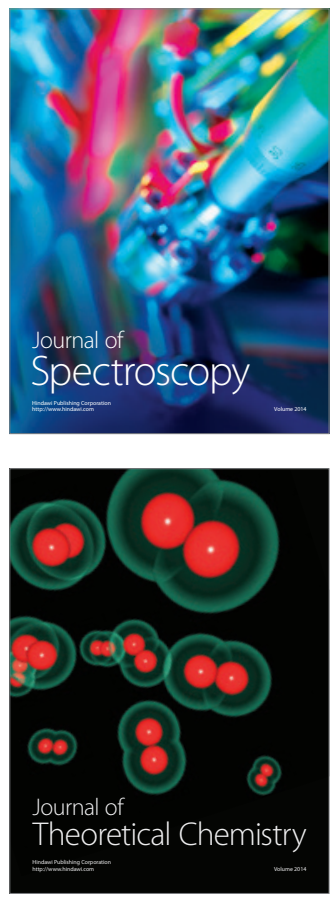
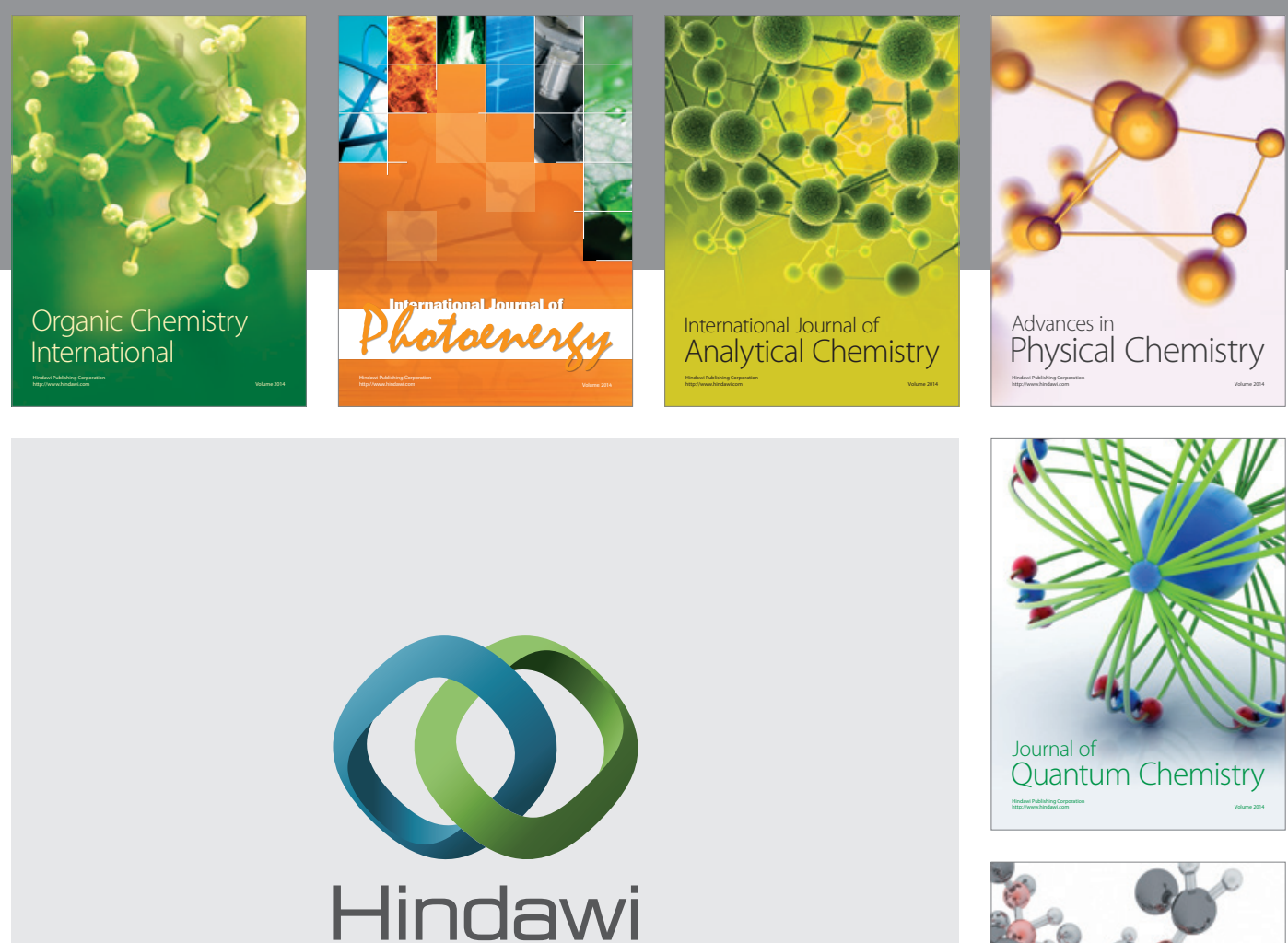

Submit your manuscripts at

http://www.hindawi.com

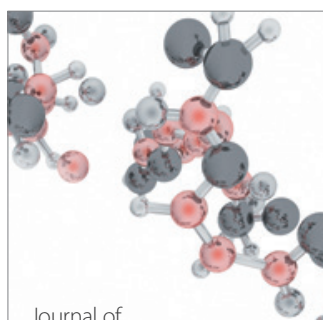

Analytical Methods

in Chemistry

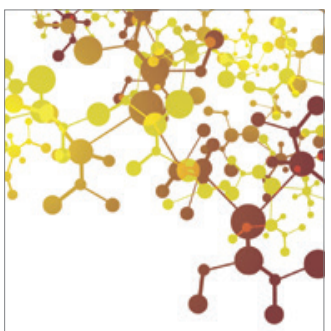

Journal of

Applied Chemistry

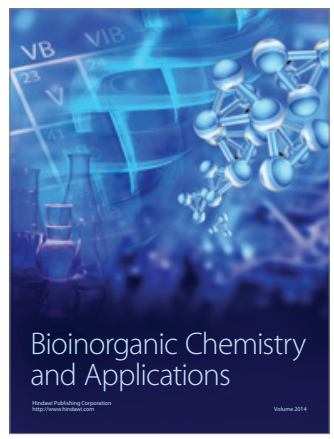

Inorganic Chemistry
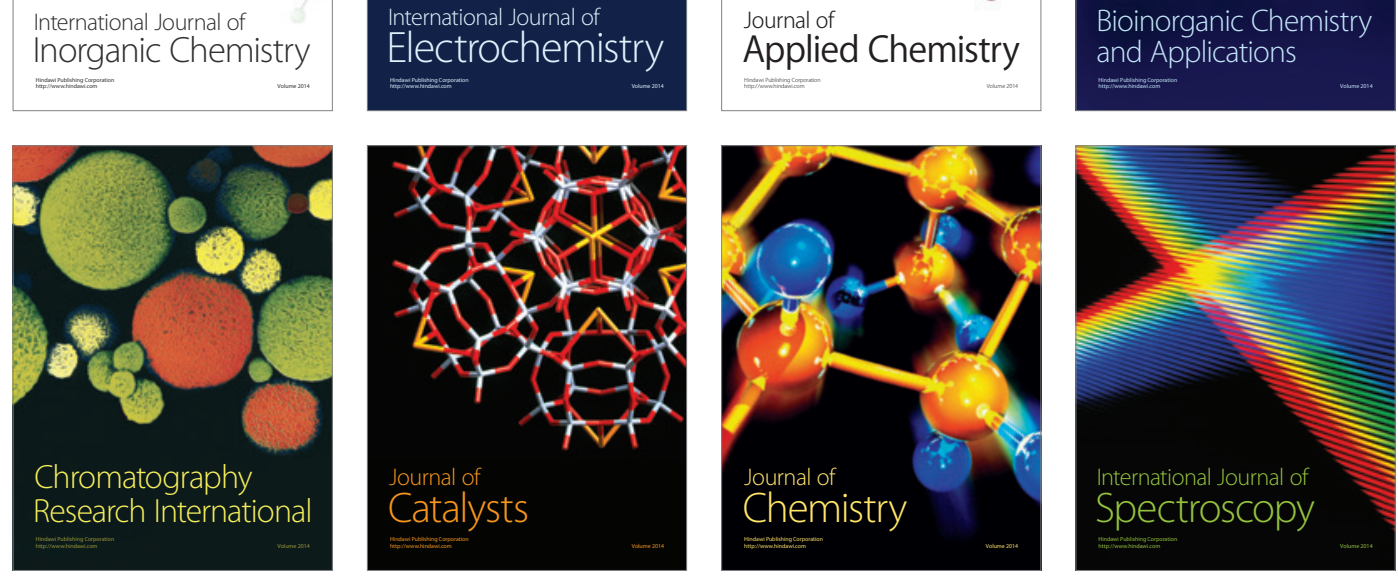